Publ. RIMS, Kyoto Univ.

15 (1979), 315-337

\title{
On Cauchy-Kowalevski's Theorem for General Systems
}

By

\author{
Masatake MIYAKE*
}

\section{$\S 1$. Introduction and Results}

Let

$$
\partial_{t}^{n_{i}} w_{i}=\sum_{j=1}^{l} q_{i j}\left(x, t ; \partial_{x}, \partial_{t}\right) w_{j}+g_{i}, \quad i=1,2, \cdots, l,
$$

be a system of linear partial differential equations defined in a neighbourhood of the origin of $\boldsymbol{C}_{x}^{n} \times \mathbb{C}_{t}^{1}$. Suppose that the order of derivatives in $t$ of $q_{i j}$ are less than $n_{j}$, then by adding $\partial_{t} w_{i}, \cdots, \partial_{t}^{n_{i}-1} w_{i},(i=1,2, \cdots, l)$ as unknown functions we have following equivalent system with (1.1),

$$
\partial_{t} u_{i}=\sum_{j=1}^{N} p_{i j}\left(x, t ; \partial_{x}\right) u_{j}+f_{i}, \quad i=1,2, \cdots, N .
$$

We shall study in this article the theorem of Cauchy-Kowalevski for the system (1.2), so we shall assume that the coefficients of operators $p_{i j}$ in (1.2) are holomorphic in $\Theta \subset \mathbb{C}_{x, t}^{n+1}$, where $\Theta=\prod_{k=1}^{n}\left\{\left|x_{k}\right|<r_{k}\right\}$ $\times\left\{|t|<r_{0}\right\}$.

Now let us consider the operator $L\left(x, t ; \partial_{x}, \partial_{t}\right)$ defined by

$$
L\left(x, t ; \partial_{x}, \partial_{t}\right)=\partial_{t} I_{N}-P\left(x, t ; \partial_{x}\right),
$$

where $I_{N}$ is the identity matrix of size $N$ and $P\left(x, t ; \partial_{x}\right)=\left(p_{i j}\right)_{i, j=1,2, \ldots, N}$.

Now in order to clarify our problem, let us give a definition.

Definition 1. 1. We sav the operator $L$ defined by (1.3) is Kowalevskian at $\left(x_{0}, t_{0}\right) \in \mathcal{O}$ if there exists a unique holomorphic solution $u(x, t)$ in a neighbourhood of $\left(x_{0}, t_{0}\right)$ of the system Lu $=f(x, t)$ with Cauch' data $U(x)$ at $t=t_{0}$, where $U(x)={ }^{t}\left(U_{1}(x), \cdots, U_{N}(x)\right)$

Communicated by S. Matsuura. January 28, 1977.

* Department of Mathematics, College of General Education, Nagoya University. 
and $f(x, t)={ }^{t}\left(f_{1}(x, t), \cdots, f_{N}(x, t)\right)$ are arbitrary holomorphic functions in a neighbourhood of $x_{0} \in \mathcal{O}_{x}=\prod_{j=1}^{n}\left\{\left|x_{k}\right|<r_{k}\right\}$ and $\left(x_{0}, t_{0}\right)$ respectively.

Recently, Professor S. Mizohata [5] obtained a necessary condition for $L$ to be Kowalevskian at the origin. And the author proved that in the case of $N=1, L$ is Kowalevskian at the origin if and only if order $P\left(x, t ; \partial_{x}\right) \leqq 1$ in $\mathcal{O}([4])$.

In the case of single equations, necessary conditions were obtained by S. Mizohata [6] and K. Kitagawa and T. Sadamatsu [3]. But we have not detailed condition for the system $L$ to be Kowalevskian. So we shall give a necessary condition for the Kowalevskian systems (see Theorem 1) and in the case of $n=1$ we shall obtain a necessary and sufficient condition (see Theorems 2 and 3).

As professor S. Mizohata pointed out in his paper, it is impossible of using the characteristis polynomial of $L$ in order to characterize the Kowalevskian systems in the case of variable coefficients. Therefore in this article, we shall apply the idea of Volevič.

Now let us remember the definition of order of operator $P\left(x, t ; \partial_{x}\right)$ $=\left(p_{i j}\right)_{i, j=1, \ldots, N}$ in the sense of Volevič. Let $r_{i j}=\operatorname{order} p_{i j}\left(x, t ; \partial_{x}\right)$ if $p_{i j} \not \equiv 0$ and $-\infty=$ order $(0)$. Then order $\left(p_{i_{j} i_{k}}\left(x, t ; \partial_{x}\right)\right)_{j, k=1, \ldots, l}$ is defined by

$$
\operatorname{order}\left(p_{i_{j} i_{k}}\left(x, t ; \partial_{x}\right)\right)_{j, k=1, \ldots, l}=\max _{\sigma \in S_{l}} \sum_{j=1}^{l} r_{i_{j} i_{\sigma(j)}},
$$

where $S_{l}$ denotes the set of permutations of $\{1,2, \cdots, l\}$ and we define $-\infty+r=-\infty$ for any $r \in \boldsymbol{Z}_{+}=\{0,1,2, \cdots, m, \cdots\}$. Now the rational number $p$ is called the order of $P\left(x, t ; \partial_{x}\right)$ in the sense of Volevič if

$$
p=\max _{\substack{1 \leq l \leq N \\ i_{1}<\cdots<i_{l}}} \frac{1}{l} \operatorname{order}\left(p_{i_{j} i_{k}}\right)_{j, k=1, \cdots, l} .
$$

In the following, order $P$ denotes the order of $P$ in the sense of Volevič. Then applying the following Lemma of Volevič, if $p=\operatorname{order} P$, then there exists a system of rational numbers $\left\{t_{i}\right\}_{i=1}^{N}$ such that

(1. 6) $\operatorname{order} p_{i j}\left(x, t ; \partial_{x}\right) \leqq t_{i}-t_{j}+p \quad$ for any $i, j=1, \cdots, N$. 
We shall say such a system of rational numbers $\left\{t_{i}\right\}_{i=1}^{N}$ an admissible system of $P$. Let $\stackrel{\circ}{p}_{i j}$ be the homogeneous part of degree $t_{i}-t_{j}+p$ of $p_{i j}$. Then $\stackrel{\circ}{P}\left(x, t ; \partial_{x}\right)=\left(\dot{p}_{i j}\right)$ is said the principal part of $P$ in Volevič's sense. Let us remark that $\stackrel{P}{P}$ depends on the choice of admissible system, but its characteristic polynomial does not depend on the choice of admissible system.

Lemma of Volevič. Suppose that $\left(r_{i j}\right)_{i, j=1, \ldots, N}$, where $r_{i j}$ $\in Z^{\cup}\{-\infty\} \quad$ (resp. $\in Q^{\cup}\{-\infty\}$ ) satisfies the following conditions: $\max _{\sigma \in S_{l}} \sum_{j=1}^{l} r_{i_{j} i_{\sigma(j)}} \leqq 0$ for any $i_{1}<\cdots<i_{l}$ and $l=1, \cdots, N$. Then there exists a system of integers $\left\{t_{i}\right\}_{i=1}^{N}$ (resp. of rational numbers) satisfying $r_{i j}$ $\leqq t_{i}-t_{j}$ for any $i, j=1,2, \cdots, N$.

In fact, such a system of numbers is obtained as follows: Let $t_{1} \in \mathbb{Z}$ (resp. $\in \boldsymbol{Q}$ ) be given arbitrary. Then $t_{j},(j=2, \cdots, N)$ are obtained inductively as follows,

$$
\begin{aligned}
& t_{j} \in\left[\max _{1 \leqq k \leqq j-1}\left\{r_{j j_{1}}+r_{j_{1} j_{2}}+\cdots+r_{j_{m} k}+t_{k}\right\},\right. \\
&\left.\min _{1 \leqq i \leqq k-1}\left\{t_{l}-r_{l i_{n}}+r_{i_{n} i_{n-1}}-\cdots-r_{i_{1} j}\right\}\right],
\end{aligned}
$$

$j=2, \cdots, N$, where $\left\{j_{1}, \cdots, j_{m}\right\},\left\{i_{1}, \cdots, i_{n}\right\} \subset\{j, j+1, \cdots, N\}$.

Definition 1. 2. We say the system $L$ is Kowalerskian in Volevič's sense if order $P \leqq 1$. In this case there exists an admissible system of integers $\left\{t_{i}\right\}_{i=1}^{N}$ of $P$, i.e., order $p_{i j} \leqq t_{i}-t_{j}+1$ for any $i, j=1,2, \cdots, N$.

Next, remember that the characteristic polynomial $p(x, t ; \zeta, \lambda)$ of $L$ is defined by

$$
p(x, t ; \zeta, \lambda)=\operatorname{det} L(x, t ; \zeta, \lambda), \quad \zeta \in \mathbb{C}^{n}, \quad \lambda \in \mathbb{C}^{1} .
$$

Definition 1. 3. Let $p(x, t ; \zeta, \lambda)=\lambda^{N}-\sum_{j=1}^{N} a_{j}(x, t ; \zeta) \lambda^{N-j}$. Then $q(\in Q)$ is said the weight of $p$ if

$$
q=\max _{1 \leqq j \leqq N}\left\{\operatorname{deg} a_{j}(x, t ; \zeta) / j\right\}
$$

where $\operatorname{deg} a_{j}$ denotes the degree of polynomial $a_{j}$ in $\zeta$. And we say that $p$ is Kowalevskian polynomial if $q \leqq 1$. 
By definitions of order $P$ and the weight $q$ of characteristic polynomial of $L$, it follows immediately that order $P \geqq q$.

Let us now remark that in the case of constant coefficients the system $L$ is Kowalevskian if and only if the characteristic polynomial of $L$ is Kowalevskian polynomial. And it is the classical result that if $L$ is Kowalevskian system in Volevič's sense, then $L$ is Kowalevskian at every point in $\mathcal{O}$, (see Gårding [1]). But, as S. Mizohata pointed out in his paper [5], it does not always follow that Kowalevskian system is the one in Volevič's sense in the case of variable coefficients. And he gave a necessary condition for $L$ to be Kowalevskian at the origin, that is,

"Let order $P>1$ and let $\stackrel{\circ}{P}\left(x, t ; \partial_{x}\right)$ be the principal part of $P$ in Volevičs sense. Then if the system $L$ is Kowalevskian at the origin, it is necessary that all the characteristic roots of $\stackrel{\circ}{P}(x, 0 ; \zeta)$ are zero for any $(x, \zeta) \in \mathcal{O}_{x} \times \mathbb{C}^{n}$."

Next theorem is an extension of that of S. Mizohata.

Theorem 1. Let $\operatorname{order} P=p>1$ and let $T=\left\{t_{i}\right\}_{i=1}^{N} \subset Q$ be an admissible system of $P$. And assume the following conditions: (i) $\stackrel{\circ}{P}(x, t ; \zeta)^{k} \not \equiv 0, \quad(k=1,2 \cdots, s-1), \stackrel{\circ}{P}(x, t ; \zeta)^{s} \equiv 0$, where $\stackrel{\circ}{P}$ is the principal part in Volevič's sense.

(ii) Let $P\left(x, t ; \partial_{x}\right)^{s}=\left(p_{i j}^{(s)}\right)$. We assume that order $p_{i j}^{(s)} \leqq t_{i}-t_{j}+s p-c$, where $s p-c=p_{s}>s$ and $0<c<p,(c \in Q)$.

Now let $\stackrel{\circ}{P}_{s}\left(x, t ; \partial_{x}\right)=\left(\check{p}_{i j}^{(s)}\left(x, t ; \partial_{x}\right)\right)$, where $\stackrel{\circ}{p}(s)_{i j}$ is the homogeneous part of degree $t_{i}-t_{j}+p_{s}$ of $p_{i j}^{(s)}$. Then if the system $L$ is Kowalevskian at the origin, it is necessary that all the characteristic roots of $\stackrel{\circ}{P}_{s}(x, 0 ; \zeta)$ are zero for any $(x, \zeta) \in \mathcal{O}_{x} \times \mathbb{C}^{n}$.

Now let us remark that from the proof of this Theorem, we can see that if order $P\left(P+\partial_{t}\right)^{m-1} \leqq m$ for some $m$ ( $\geqq 2$ ) (see Definition 2.1), then the system $L$ is Kowalevskian at every point in $\mathcal{O}$. But, we have an example ${ }^{1)}$ of Kowalevskian system which satisfies that order $P\left(P+\partial_{t}\right)>2$ and its principal part is not nilpotent. This shows that it is difficult to obtain a necessary and sufficient condition by the construction of the 
formal solution of the Cauchy problem (for more detail, see section 2). On the other hand, in the case where $P=P\left(x ; \partial_{x}\right)$ we can see that under the condition that order $P\left(x ; \partial_{x}\right)^{m}>m$ for any $m$, it is necessary that all the characteristic roots of $\stackrel{\circ}{P}_{m}(x ; \zeta)$ are zero for any $(x, \zeta)$ and any $m$, where $\stackrel{\circ}{P}_{m}$ denotes the principal part of $P^{m}$.

Next, let us consider the case of $n=1$. Then in this case we can obtain a necessary and sufficient condition. In fact, we have

Theorem 2. In the case $n=1$, the system $L$ is Korvalevskian at every point in $\mathcal{O}$ if and only if there exists an operator $J\left(x, t ; \partial_{x}\right)$ of $N \times N$ matrix satisfying the following conditions:

(i) The coefficients of $J$ are meromorphic functions in $\mathcal{O}$.

(ii) $J$ is invertible, that is, there exists an operator $J^{-1}\left(x, t ; \partial_{x}\right)$ such that $J J^{-1}=J J^{-1}=I_{N}$, where $I_{N}$ denotes the identity matrix of size $N$.

(iii) $J^{-1} L J$ is Korvalevskian system in Volevičs sense.

Theorem 2 asserts that the notions of Kowalevskian system and the one in Volevič's sense are the same essentially. But the notion of the Kowalevskian system is more closely connected with the matrix structure than the order relation between $p_{i j}$. In fact, there exists an example that $\partial_{t} I_{2}-P\left(t ; \partial_{x}\right)$ is Kowalevskian but $\partial_{t} I_{2}+P\left(t ; \partial_{x}\right)$ is not Kowalevskian. For this purpose it suffices to choose $P\left(t ; \partial_{x}\right)$ given by the footnote 1 . Such a phenomenon does not appear if we consider only on order relation between $p_{i j}$.

Now let us consider the case of constant coefficients. In this case we can express the condition in another way as follows: The system $L$ is Kowalevskian if and only if

1) $L\left(t ; \partial_{x}, \partial_{t}\right)=\partial_{t} I_{2}-P\left(t ; \partial_{x}\right)$, where $P\left(t ; \partial_{x}\right)=\left[\begin{array}{rr}t \partial_{x}^{3} & \partial_{x}^{2} \\ -t^{2} \partial_{x}^{4} & -t \partial_{x}^{s}\end{array}\right]-\left[\begin{array}{ll}0 & 0 \\ \partial_{x} & 0\end{array}\right]$. Let $J\left(t ; \partial_{x}\right)$ $=\left[\begin{array}{cc}1 & 0 \\ -t \partial_{x} & 1\end{array}\right]$. Then we can see that $J^{-1} L J$ is Kowalevskian in Volevičs sense. On the other hand, we can prove that

$$
P\left(P+\partial_{t}\right)=-2\left[\begin{array}{cc}
0 & 0 \\
t \partial_{x}^{4} & \partial_{x}^{3}
\end{array}\right]
$$




$$
\lim _{m \rightarrow \infty} \operatorname{order} P\left(\partial_{x}\right)^{m} / m \leqq 1
$$

In fact, we can prove,

Proposition 1. Let $p(\zeta, \lambda)=\lambda^{N}-\sum_{j=1}^{N} a_{j}(\zeta) \lambda^{N-j}$ be the characteristic polynomial of $L$ and let $q$ be the reight of $p(\zeta, \lambda)$. Then we have

$$
\lim \operatorname{order} P\left(\partial_{x}\right)^{m} / m=q
$$

Hence, this Proposition suggests that in the case of $P=P\left(x ; \partial_{x}\right)$, the system $L$ is Kowalevskian if and only if

$$
\limsup _{m \rightarrow \infty} \operatorname{order} P\left(x ; \partial_{x}\right)^{m} / m \leqq 1
$$

But we can prove it only in the case $n=1$.

Theorem 3. In the case of $n=1$ and $P=P\left(x ; \partial_{x}\right)$, the system $L$ is Kowalevskian at the origin if and only if the condition (1.12) is valid. More pricisely, let

$$
\lim \text { sup order } P\left(x ; \partial_{x}\right)^{m} / m=q \geqq 0 .
$$

Then there exists an operator $J\left(x ; \partial_{x}\right)$ of $N \times N$ matrix satisfying the conditions (i) and (ii) in Theorem 2 and if we put $\widetilde{P}_{0}\left(x ; \partial_{x}\right)$ as the principal part of $J^{-1} P J$, then it holds that order $J^{-1} P J=q$ and $\widetilde{P}_{0}\left(x_{0}, \zeta_{0}\right)$ has non-zero characteristic roots for some $\left(x_{0}, \zeta_{0}\right) \in \mathcal{O}_{x} \times \boldsymbol{C}^{1}$.

At the end we shall give a local uniqueness theorem.

Theorem 4. (Holmgren) Let $(x, t) \in \boldsymbol{R}^{n+1}$. If there exists an operator $J\left(x, t ; \partial_{x}\right)$ satisfying the conditions (i), (ii) and (iii) in Theorem 2, the local uniqueness theorem holds for the Cauchy problem to $L$. Pricisely, let $u(x, t) \in C^{1}\left([-T, T] ; \mathscr{D}^{\prime}\left(\Omega_{x}\right)\right)$ satisfy $L u=0$ and $u(x, 0)=0$, where $0 \in \Omega_{x}$. Then $u(x, t)$ vanishes in a neighbourhood of the origin.

We should remark that in the case where the coefficients of $J$ and 
$J^{-1}$ are real analytic at the origin, this theorem is trivial. Therefore our interest lies in the case where the coefficients of $J$ or $J^{-1}$ are singular at the origin.

This article is constructed as follows. In section 2 we shall prove Theorem 1. Section 3 is devoted to the proof of Theorems 2 and 3. And at the end of Section 3 , we shall give a system defined in $\boldsymbol{C}_{x, t}^{n+1},(n \geqq 2)$ which corresponds to Theorems 2 and 3 but is not proved by our method. Finally Theorem 4 will be proved in Section 4 .

\section{§2. Proof of Theorem 1 .}

In general, the domain of existence of the solution of the Cauchy problem depends on Cauchy data, but concerning this we have

Lemma 2. 1. (S. Mizohata [5]). Let L be Kowalevskian at the origin and let $H\left(\Omega_{x}\right)$ be the set of holomorphic functions in $\Omega_{x}$, where $0 \in \Omega_{x} \subset \mathcal{O}_{x}$. Then there exists $D(\subset \mathcal{O})$ which depends only on $\Omega_{x}$ such that the system $L u=0$ has holomorphic solution $u \in H(D)$ with Cauchy data $\left.u\right|_{t=0}=U(x) \in H\left(\Omega_{x}\right)$.

Definition 2. 1. $P\left(P+\partial_{t}\right)$ denotes the operator defined by $P\left(P+\partial_{t}\right)=P^{2}+P_{t}$, where $P_{t}$ is the operator obtained by differentiating by $t$ the coefficients of $P$. In general, $P\left(P+\partial_{t}\right)^{m}=\left(P\left(P+\partial_{t}\right)^{m-1}\right) P$ $+\left(P\left(P+\partial_{t}\right)^{m-1}\right)_{t}$.

Now let us consider the following Cauchy problem,

$$
\begin{aligned}
& L u=0, \\
& \left.u\right|_{t=0}=U(x),
\end{aligned}
$$

where $L=\partial_{t} I_{N}-P\left(x, t ; \partial_{x}\right)$. Let $u(x, t) \sim \sum_{m \geq 0} u_{m}(x) t^{m} / m$ ! be the formal solution of $(2.1)-(2.2)$. Then by definition of $P\left(P+\partial_{t}\right)^{m},(m \geqq 1)$ we have

$$
u_{m}(x)=\left[P\left(P+\partial_{t}\right)^{m-1}\right]_{t=0} U(x), \quad(m \geqq 1),
$$

where $P\left(P+\partial_{t}\right)^{0}=P$. 
In order to investigate $u_{m}(x)$, we prepare the following two lemmas.

Lemma 2. 2. $P\left(P+\partial_{t}\right)^{m-1}$ is expressed as follows:

$$
P\left(P+\partial_{t}\right)^{m-1}=P^{m}+\sum_{k=1}^{m}\left(P^{m-k}\right){ }_{t} P^{k-1}+R_{m-1}\left(x, t ; \partial_{x}\right),
$$

where $R_{m-1}$ is a linear sum of each term which is a product at most $m-2$ terms in $\left\{P, P_{t}, \cdots, P_{m-1}^{P_{t \ldots t}}\right\}$.

This is a result of an elementary calculation.

Now we put

$$
P\left(P+\partial_{t}\right)^{m-1}=P^{m}+Q_{m-1}+R_{m-1} .
$$

For the simplicity of the discription, in the following $\underset{T}{\operatorname{order}} P \leqq p$ means order $p_{i j} \leqq t_{i}-t_{j}+p$ for any $i, j$, where $T=\left\{t_{i}\right\}_{i=1}^{N} \subset Q$.

Lemma 2. 3. Suppose the conditions in Theorem 1. Then we have

$$
\begin{aligned}
& \underset{T}{\operatorname{order}} P^{s k} \leqq k p_{s}, \\
& \underset{T}{\operatorname{order}} Q_{s k-1} \leqq k p_{s}-(p-c), \\
& \underset{T}{\operatorname{order}} R_{s k-1} \leqq k p_{s}-2(p-c) .
\end{aligned}
$$

Proof. (i) is evident. (ii): Let us consider each term $\left(P^{l}\right)_{t} P^{s k-l-1}$, $(l=1, \cdots, s k-1)$. Put $l=a s+q$ and $s k-l-1=b s+r$. Then $a+b=k$ -1 and $q+r=s-1$. On the other hand, we have $\underset{T}{\operatorname{order}} P^{a s+q} \leqq a p_{s}+q p$, which implies immediately our result.

(iii): Let us prove it by induction on $k$. It is evident when $k=1$, since $(s-2) p<p_{s}-2(p-c)$. Let (iii) be valid up to $k$. Now we shall examine the construction of $R_{s(k+1)-1}$.

$$
\begin{aligned}
P\left(P+\partial_{t}\right)^{s k}=\left(P^{s k}+Q_{s k-1}+R_{s k-1}\right) P+\left(P^{s k}+Q_{s k-1}+R_{s k-1}\right)_{t} \\
\quad=P^{s k+1}+\left(P^{s k}\right)_{t}+Q_{s k-1} P+R_{s k-1} P+\left(Q_{s k-1}\right)_{t}+\left(R_{s k-1}\right)_{t} \\
\quad=P^{s k+1}+Q_{s k}+\left\{R_{s k-1} P+\left(Q_{s k-1}\right)_{t}\right\}+\widetilde{R}_{s k}
\end{aligned}
$$

where $Q_{s k}=\left(P^{s k}\right)_{t}+Q_{s k-1} P$ and $\widetilde{R}_{s k}=\left(R_{s k-1}\right)_{t}$. By the assumption of 
induction, we have $\underset{T}{\operatorname{order}} \widetilde{R}_{s k} \leqq k p_{s}-2(p-c)$. In general, we have

$$
\begin{aligned}
& P\left(P+\partial_{t}\right)^{s k+l}=P^{s k+l+1}+Q_{s k+l} \\
& \quad+\left\{R_{s k-1} P^{l+1}+\sum_{j=0}^{l}\left(Q_{s k+j-1}\right)_{t} P^{l-j}\right\}+\widetilde{R}_{s k+l},
\end{aligned}
$$

where

$$
\begin{aligned}
Q_{s k+l}= & \left(P^{s k+l}\right)_{t}+\left(Q_{s k+l-1}\right) P, \\
\widetilde{R}_{s k+l}= & \widetilde{R}_{s k+l-1} P+\left(\widetilde{R}_{s k+l-1}\right)_{t} \\
& +\left\{R_{s k-1} P^{l}+\sum_{j=0}^{l-1}\left(Q_{s k+j-1}\right)_{t} P^{l-1-j}\right\}_{t} .
\end{aligned}
$$

We can prove by induction on $l$ that order $\widetilde{R}_{s k+l} \leqq k p_{s}+l p-2(p-c)$. In fact, it suffices to see that order $Q_{s k+l} \leqq k p_{s}+l p+c$, which can be proved easily by induction on $l,(l=-1,0, \cdots)$. Hence, by putting $l=s-1$ we have order $\widetilde{R}_{(k+1) s-1}<(k+1) p_{s}-2(p-c)$. On the other hand, we have order $R_{s k-1} P^{s} \leqq(k+1) p_{s}-2(p-c)$, and $\underset{T}{\operatorname{order}}\left(Q_{s k+j-1}\right)_{t} P^{s-1-j}$ $\leqq k p_{s}+(j-1) p+c+(s-1-j) p=(k+1) p_{s}-2(p-c),(j=0,1, \cdots, s-1)$, which implies (iii).

Q.E.D.

Proof of Theorem 1. Remember that the formal solution $u(x, t)$ $\sim \sum_{m \geqq 0} u_{m}(x) t^{m} / m$ ! of the Cauchy problem (2.1)-(2.2) is given by

$$
u_{m}(x)=\left\{P^{m}\left(x, 0 ; \partial_{x}\right)+Q_{m-1}\left(x, 0 ; \partial_{x}\right)+R_{m-1}\left(x, 0 ; \partial_{x}\right)\right\} U(x),
$$

$m \geqq 0$. In the following, we shall consider such $k$ as

$$
k p_{s}=\text { integer }
$$

Therefore, we have

$$
u_{s k}(x)=\left\{P^{s k}\left(x, 0 ; \partial_{x}\right)+Q_{s k-1}\left(x, 0 ; \partial_{x}\right)+R_{s k-1}\left(x, 0 ; \partial_{x}\right)\right\} U(x) .
$$

We shall prove the theorem by the contradiction. We assume that there exists $\left(x_{0}, t_{0}\right) \in \mathcal{O}_{x} \times C^{n}$ such that there exists a non-zero characteristic value $\lambda\left(x_{0}, t_{0}\right)$ of $\stackrel{\circ}{P}_{s}\left(x_{0}, t_{0} ; \zeta_{0}\right)$.

First, we assume that $\stackrel{\circ}{P}_{s}\left(0,0 ; \zeta_{0}\right)$ has a non-zero characteristic value $\lambda$. Now we put

$$
P\left(x, 0 ; \partial_{x}\right)^{s}=\stackrel{\circ}{P}_{s}\left(0,0 ; \partial_{x}\right)+P_{s, 1}\left(x ; \partial_{x}\right),
$$


where order $P_{s, 1}\left(x ; \partial_{x}\right) \leqq p_{s}$ and its homogeneous part of order $p_{s}$ in the sense of Volevič vanishes at the origin. Then we have

$$
u_{s k}(x)=\stackrel{\circ}{P}_{s}\left(\partial_{x}\right)^{k} U(x)+P_{s k}\left(x ; \partial_{x}\right) U(x),
$$

where $\stackrel{\circ}{P}_{s}\left(\partial_{x}\right)=\stackrel{\circ}{P}_{s}\left(0,0 ; \partial_{x}\right)$ and order $P_{s k} \leqq k p_{s}$ and its homogeneous part of order $k p_{s}$ in the sense of Volevič vanishes at the origin.

Let $\xi \in C^{N}$ be the eigen vector corresponding to $\lambda$, i.e., $\stackrel{\circ}{P}_{s}\left(\zeta_{0}\right) \xi=\lambda \xi$. We put $\stackrel{\circ}{P}_{s}\left(\zeta_{0}\right)^{k}=\left(\dot{p}_{i j}^{(s k)}\right)$. Then $\sum \dot{p}_{i j}^{(s k)} \xi_{j}=\lambda^{k} \xi_{i}, i=1, \cdots, N$. Considering that order $\stackrel{p}{p}_{i j}^{(s k)}\left(\partial_{x}\right)=t_{i}-t_{j}+k p_{s}$, where $k p_{s}=$ integer, if we give $\hat{\xi}^{\prime} \in \boldsymbol{C}^{N}$ by

$$
\xi^{\prime}={ }^{t}\left(\xi_{1}^{\prime}, \cdots, \xi_{N}^{\prime}\right), \quad \xi_{i}^{\prime}= \begin{cases}\xi_{i} & \text { if } t_{i} \text { is an integer } \\ 0 & \text { otherwise }\end{cases}
$$

then we have

$$
\sum_{j} \stackrel{\circ}{p}_{i j}^{(s k)}\left(\zeta_{0}\right) \xi_{j}^{\prime}=\lambda^{k} \xi_{i}^{\prime}, \quad i=1, \cdots, N
$$

Now we may assume without loss of generality that

$$
\xi_{i_{0}}^{\prime} \neq 0, \quad t_{i_{0}}=\text { integer and } t_{i} \leqq 0 \text { for any } i \text {. }
$$

Let $U(x)={ }^{t}\left(U_{1}(x), \cdots, U_{N}(x)\right)$ be the Cauchy data defined by

$$
U_{i}(x)=\sum_{k}^{\prime} e^{i \theta_{k}}\left(k p_{s}\right) ! \frac{\left\langle\zeta_{0}, x\right\rangle^{k p_{s}-t_{i}}}{\left(k p_{s}-t_{i}\right) !} \xi_{i}^{\prime},
$$

where $\sum_{k}^{\prime}$ denotes the summation orer $k$ such as $k p_{s}=$ integer. Let $u_{s k, i_{0}}(x)$ be the $i_{0}$-th component of $u_{s k}$. Then we have

$$
\left.u_{s k, i_{0}}(x)=\sum_{j}{\stackrel{\circ}{i_{0} j}(s k)}_{\left(\partial_{x}\right.}\right) U_{j}(x)+\sum_{j} p_{i_{0} j}^{(s k)}\left(x ; \partial_{x}\right) U_{j}(x),
$$

where order ${\stackrel{p}{i_{0} j}}^{(s k)}\left(\partial_{x}\right)=t_{i_{0}}-t_{j}+k p_{s}$, order $p_{i_{0} j}^{(s k)} \leqq t_{i_{0}}-t_{j}+k p_{s}$ and its homogeneous part of order $t_{i_{0}}-t_{j}+k p_{s}$ vanishes at the origin. Hence, we have

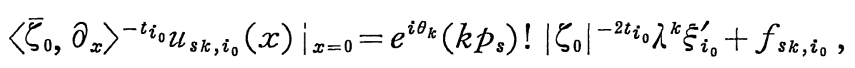

where $f_{s k, i_{0}}$ is a constant depending only on $\theta_{0}, \cdots, \theta_{k-1}$. Now we put

$$
\theta_{k}=\arg f_{s k, i_{0}}-\arg \lambda^{k} \xi_{i_{0}}^{\prime}, \quad k \geqq 1,
$$

where $\theta_{0}$ is given arbitrary. Then we have

$$
\left|\left[\left\langle\bar{\zeta}_{0}, \partial_{x}\right\rangle^{-t_{i_{0}} u_{s k, i_{0}}}(x)\right]_{x=0}\right| \geqq\left(k p_{s}\right) !|\lambda|^{k}\left|\xi_{i_{0}}^{\prime}\right|\left|\zeta_{0}\right|^{-2 t_{i_{0}}} .
$$


On the other hand, we know that $p_{s}>s$ by the condition in Theorem 1, which implies that the formal solution constructed in the above is not holomorphic in any neighbourhood of the origin. Therefore, it is necessary that all the characteristic values of $\stackrel{\circ}{P}_{s}(0,0 ; \zeta)$ are zero for any $\zeta \in \mathbb{C}^{n}$.

In the case that all the characteristic values of $\dot{P}_{s}(0,0 ; \zeta)$ are zero for any $\zeta$, but there exists $\left(x_{0}, \zeta_{0}\right) \in \mathcal{O}_{x} \times C^{n}$ such that $\stackrel{\circ}{P}_{s}\left(x_{0}, 0 ; \zeta_{0}\right)$ has non-zero characteristic values, we may assume that $x_{0}$ is as near the origin as we need. In view of Lemma 2.1 , if the system $L$ is Kowalevskian at the origin, then there exists the solution $u \in H(D)$ of the Cauchy problem $L u=0,\left.u\right|_{t=0}=U(x) \in H\left(\Theta_{x}\right)$, where $D$ depends only on $\mathcal{O}_{x}$. Let $x_{0} \in D_{\cap}\{t=0\}$. Then we can construct the Cauchy data in $H\left(\mathcal{O}_{x}\right)$ such that the Cauchy problem $L u=0,\left.u\right|_{t=0}=U(x)$ has not holomorphic solution in any neighbourhood of $\left(x_{0}, 0\right)$, which contradicts the assumption that $L$ is Kowalevskian at the origin.

Q.E.D.

\section{$\S 3 . \quad$ Proof of Theorems 2 and 3}

In order to prove Theorem 2, we prepare some lemmas.

Lemma 3. 1. Let $L=\partial_{t}-P$ be the system given by (1.3) and let $n=1$. Suppose that the all the characteristic values of $\stackrel{\circ}{P}(x, t ; \zeta)$ are zero for any $(x, t, \zeta) \in \mathcal{O} \times \mathbb{C}^{1}$, then there exists an operator $J\left(x, t ; \partial_{x}\right)$ with properties (i) and (ii) in Theorem 2. And also, if we put $J^{-1} L J=\partial_{t} I_{N}-\widetilde{P}$, then order $\widetilde{P} \leqq$ order $P-\varepsilon(N)$, where $\varepsilon(N)$ is a positive constant depending only on $N$.

Proof. Let $\stackrel{\circ}{p}_{i}\left(x, t ; \partial_{x}\right)=\left(\stackrel{\circ}{p}_{i 1}, \cdots, \stackrel{\circ}{p}_{i N}\right)$ and $\stackrel{\circ}{p}^{j}\left(x, t ; \partial_{x}\right)={ }^{t}\left(\stackrel{\circ}{p}_{1 j}, \cdots, \stackrel{\circ}{p}_{N j}\right)$ be the $i$-th row and $j$-th column vector of $\stackrel{\circ}{P}$ respectively, where $\stackrel{\circ}{P}$ is the principal part of $P$ in Volevič's sense. Then if ${\stackrel{\circ}{p_{0}}}_{i_{0}} \equiv 0$ (resp. $\stackrel{\circ}{p}^{j_{0}} \equiv 0$ ), we can regard that $\not^{i_{0}} \equiv 0$ (resp. ${\stackrel{\circ}{j_{0}}} \equiv 0$ ) by a suitable choice of admissible system. In fact, ${\stackrel{\circ}{i_{0}}}_{i_{0}} \equiv 0$ implies that order ${\stackrel{\circ}{i_{0} j}}_{i_{0}}<t_{i_{0}}-t_{j}+p$, where order $P$ $=p$ and $T=\left\{t_{i}\right\}_{i=1}^{N}$ is an admissible system. Hence, there exists $r>0$ $(r \in Q)$ such that order $p_{i_{0} j}<t_{i_{0}}-\left(t_{j}+r\right)+p$ if $j \neq i_{0}$. Now it is easy to see that $S=\left\{s_{i}\right\}$, where $s_{i}=t_{i}+r$ if $i \neq i_{0}$ and $s_{i_{0}}=t_{i_{0}}$, is also an admissible system of $P$, and we have for this admissible system ${\stackrel{\circ}{i_{0}}} \equiv{\stackrel{\circ}{p_{0}}}^{i_{0}}$ 
$\equiv 0$. Therefore, without loss of generality we may assume that $\stackrel{\circ}{i j}_{i j} \equiv 0$ if $i \geqq k+1$ and $j \geqq k+1$, and $t_{1} \leqq t_{2} \leqq \cdots \leqq t_{k}$ by a change of row and column if necessary. Obviously we assume that $\stackrel{\circ}{p}_{i} \not \equiv 0$ and $\stackrel{\circ}{p}^{j} \not \equiv 0$ if $i, j \leqq k$.

Since rank $\stackrel{\circ}{P}(x, t ; \zeta) \leqq k-1$, there exists a left null vector of $\stackrel{\circ}{P}(x, t ; \zeta)$ of the form

$$
l(x, t ; \zeta)=\left(l_{1} \zeta^{t_{i_{0}-t_{1}}}, \cdots, l_{i_{0}-1} \zeta^{t_{i_{0}-t_{i_{0}-1}}}, 1,0, \cdots, 0\right),
$$

where $2 \leqq i_{0} \leqq k$ and $l_{i}(x, t)$ are meromorphic functions in $\mathcal{O}$. Without loss of generality, we may assume that $l_{i} \equiv 0$ if $t_{i_{0}}-t_{i} \notin Z_{+}=\{0,1,2, \cdots\}$. In fact, we can easily see the following:

(i) If $t_{i_{0}}-t_{j}+p \in Z_{+}$, then $\stackrel{\circ}{p}_{i j} \not \equiv 0$ implies $t_{i_{0}}-t_{i} \in Z_{+}$, where $i=1, \cdots$, $i_{0}-1$.

(ii) If $t_{i_{0}}-t_{j}+p<0$, then $\stackrel{\circ}{p}_{i j} \equiv 0$ for any $i=1, \cdots, i_{0}-1$.

(iii) If $t_{i_{0}}-t_{j}+p \notin \mathbb{Z}_{+\cap} \mathbb{R}_{+},\left(\boldsymbol{R}_{+}=\{x ; x>0\}\right)$, then $\stackrel{\circ}{p}_{i j} \equiv 0$ if $t_{i_{0}}-t_{i} \in \boldsymbol{Z}_{+}$, $i=1, \cdots, i_{0}-1$.

Now we put

$$
\left.\tilde{J}\left(x, t ; \partial_{x}\right)^{-1}=i_{0}\right)\left(\begin{array}{rrrrr}
1 & & & \\
& \ddots & 0 & & \\
& \ddots & & \\
l_{1} \partial_{x}^{t_{i_{0}}-t_{1}} \cdots & & & \\
& & & \ddots & \\
0 & & & \ddots & \\
& & & &
\end{array}\right) .
$$

Then we have

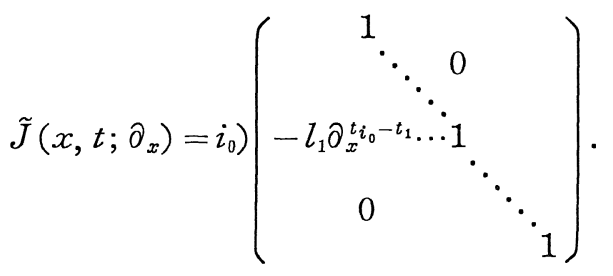

Let $\tilde{J}^{-1} L \tilde{J}=\partial_{t} I_{N}-\widetilde{P}$. Then since $\widetilde{P}=\tilde{J}^{-1} P \tilde{J}-\tilde{J}^{-1} \tilde{J}_{t}$ and $\tilde{J}^{-1} \tilde{J}_{t}=\tilde{J}_{t}$, it is obvious that order $\widetilde{P} \leqq \operatorname{order} P$. If order $\widetilde{P}=\operatorname{order} P$, then the principal part $\widetilde{P}_{0}$ of $\widetilde{P}$ with respect to the admissible system $T$ is $\widetilde{P}_{0}(x, t ; \zeta)$ $=\tilde{J}^{-1}(x, t ; \zeta) P(x, t ; \zeta) \tilde{J}(x, t ; \zeta)$. Hence, $\widetilde{P}_{0}(x, t ; \zeta)$ is nilpotent for any $(x, t, \zeta)$ and order $p_{i_{0} j}<t_{i_{0}}-t_{j}+p,(j=1,2, \cdots, N)$. By the above operations, we can obtain an invertible operator $J\left(x, t ; \partial_{x}\right)$ of $N \times N$ matrix with meromorphic coefficients such that if we put $J^{-1} L J=\partial_{t} I_{N}-\widetilde{P}$, 
then order $\widetilde{P}<$ order $P$.

At the end, we should remark that if $\operatorname{order} Q<\operatorname{order} P$, then there exists a positive constant $\varepsilon(N)$, which depends only on the size $N$ of matrix $P$, such that order $Q \leqq$ order $P-\varepsilon(N)$.

Q.E.D.

Proof of the Necessity in Theorem 2. Now we assume that $L$ is Kowalevskian at every point in $\mathcal{O}$ and $\operatorname{order} P=p>1$. Then $\stackrel{\circ}{P}(x, t ; \zeta)$ should be nilpotent matrix for any $(x, t, \zeta)$. Therefore, by Lemma 3.1, there exists an operator $J\left(x, t ; \partial_{x}\right)$ satisfying the properties in Lemma 3.1. It is obvious that if $L$ is Kowalevskian at every point in $\mathcal{O}$, then the system $J^{-1} L J$ is also Kowalevskian in the domain where the coefficients of $J$ and $J^{-1}$ are holomorphic. Therefore if $J^{-1} L J$ is not Kowalevskian in Volevič's sense, we continue the above procedure.

Q.E.D.

In order to prove the sufficient condition, we need some preparations. First, we should remark properties concerning solutions of Cauchy problem for Kowalevskian systems in Volevič's sense.

Let us consider the following Cauchy problem,

$$
\begin{aligned}
& L\left(x, t ; \partial_{x}, \partial_{t}\right) u=f\left(x, t ; x_{0}, t_{0}\right), \\
& \left.u\right|_{t=t_{0}}=U\left(x ; x_{0}\right),
\end{aligned}
$$

where $L$ is Kowalevskian system in Volevič's sense, $f$ (resp. $U$ ) is holomorpic function in a neighbourhood of $\left(x_{0}, t_{0}\right)$ (resp. $x_{0}$ ).

Now assume that radius of convergence of $f$ (resp. $U$ ) does not depend on parameter $\left(x_{0}, t_{0}\right)$ (resp. $\left.x_{0}\right)$ when $\left(x_{0}, t_{0}\right)$ varies in a compact set. And also assume that $f$ (resp. $U$ ) are uniformly bounded in $\left(x_{0}, t_{0}\right)$ (resp. $\left.x_{0}\right)$. Then we can prove that the radius of convergence of the solution $u\left(x, t ; x_{0}, t_{0}\right)$ of $(3.1)-(3.2)$ does not depend on $\left(x_{0}, t_{0}\right)$. Moreover we can show that solutions $u\left(x, t ; x_{0}, t_{0}\right)$ are uniformly bounded. Roughly speaking,

$$
\sup \left|u\left(x, t ; x_{0}, t_{0}\right)\right| \leqq C(A+B),
$$

where $C$ is a positive constant depending only on $L, \sup |f| \leqq A$, sup $|U| \leqq B$. We omit the proof of the above statements, since it seems obvious from the proof of the existence theorem of Cauchy-Kowalevski. 
Lemma 3.2. Let $J$ satisfy the conditions (i), (ii) and (iii) in Theorem 2. Assume that the coefficients of $J$ and $J^{-1}$ are holomorphic in

$$
\Omega=\prod_{k=1}^{n}\left\{s_{k}-\varepsilon_{k}<\left|x_{k}\right|<s_{k}+\varepsilon_{k}\right\} \times\left\{s_{0}-\varepsilon_{0}<|t|<s_{0}+\varepsilon_{0}\right\} .
$$

Let $f \in H\left(\prod_{k=1}^{n}\left\{\left|x_{k}\right|<\gamma_{k}\right\} \times\left\{|t|<\gamma_{0}\right\}\right)$ and $U \in H\left(\prod_{k=1}^{n}\left\{\left|x_{k}\right|<\gamma_{k}\right\}\right)$, where $s_{k}+\varepsilon_{k}<\gamma_{k}<r_{k}$. Then the Cauchy problem, Lu=f and $\left.u\right|_{t=0}=U$ has a holomorphic solution in a neighbourhood of the origin.

Proof. Let us consider the following Cauchy problem instead of the original one,

$$
\begin{aligned}
& L u=f, \\
& \left.u\right|_{t=t_{0}}=U(x) .
\end{aligned}
$$

Let

$$
u\left(x, t ; t_{0}\right)=\sum_{m \geqq 0} u_{m}\left(x ; t_{0}\right)\left(t-t_{0}\right)^{m} / m !
$$

be a formal solution of the above problem. Then $u_{m}\left(x, t_{0}\right)$ are holomorphic in $\prod_{j=1}^{n}\left\{\left|x_{k}\right|<\gamma_{k}\right\} \times\left\{|t|<\gamma_{0}\right\}$. In fact, $u_{0}\left(x ; t_{0}\right)=U(x), u_{1}\left(x, t_{0}\right)=P\left(x, t_{0} ; \partial_{x}\right)$ $\times U(x)+f\left(x, t_{0}\right)$ and generally there exists operators $\mathcal{L}_{j}^{(m)}\left(x, t ; \partial_{x}\right)$, $(j=0,1,2, \cdots, m-1)$ with holomorphic coefficients in $\mathcal{O}$, such that

$$
u_{m}\left(x, t_{0}\right)=P_{m}\left(x, t_{0} ; \partial_{x}\right) U(x)+\sum_{j=0}^{m-1} \mathcal{L}_{j}^{(m)}\left(x, t_{0} ; \partial_{x}\right)\left(\partial_{t}{ }^{j} f\right)\left(x, t_{0}\right),
$$

where $P_{m}=P\left(P+\partial_{t}\right)^{m-1}$ defined by Definition 2. 1 .

Our purpose is to prove that the formal solution (3.3) converges at the origin when $t_{0}=0$. Now we remark that (3.3)-(3.4) is equivalent with the following,

$$
\begin{aligned}
& \left(J^{-1} L J\right)\left(J^{-1} u\right)=J^{-1} f, \\
& \left.\left(J^{-1} u\right)\right|_{t=t_{0}}=J^{-1}\left(x, t_{0} ; \partial_{x}\right) U(x),
\end{aligned}
$$

where $J^{-1} L J$ is Kowalevskian in Volevič's sense. From the assumptions on $J$ and $J^{-1}$, and in view of the remark before Lemma 3.2, we may assume that the holomorphic solution $u\left(x, t ; t_{0}\right),\left(\left|t_{0}\right|=s_{0}\right)$ exists in 


$$
J_{t_{0}}=\prod_{k=1}^{n}\left\{s_{k}-\delta_{k} \leqq\left|x_{k}\right| \leqq s_{k}+\delta_{k}\right\} \times\left\{\left|t-t_{0}\right| \leqq \delta_{0}\right\}
$$

for some positive constants $\delta_{k}\left(\delta_{k}<\varepsilon_{k}\right)$, and also we may assume that $\left(J^{-1} u\right)\left(x, t ; t_{0}\right)$ are uniformly bounded in $J_{t_{0}}$ when $t_{0}$ varies in $\left\{\left|t_{0}\right|=s_{0}\right\}$. Let

$$
M=\sup _{J_{t},\left|t_{0}\right|=s_{0}}\left|u\left(x, t ; t_{0}\right)\right|
$$

Then by Cauchy's integral formula we have

$$
\left|u_{m}\left(x ; t_{0}\right)\right| \leqq M \cdot m ! / \delta_{0}{ }^{m},
$$

for any $x \in \prod_{k=1}^{n}\left\{s_{k}-\delta_{k} \leqq\left|x_{k}\right| \leqq s_{k}+\delta_{k}\right\}$ and $\left|t_{0}\right|=s_{0}$. Now because of that $u_{m}\left(x ; t_{0}\right)$ is holomorphic in $\prod_{k=1}^{n}\left\{\left|x_{k}\right|<\gamma_{k}\right\} \times\left\{\left|t_{0}\right|<\gamma_{0}\right\}$, we have by the maximum principle,

$$
\mid u_{m}(x ; 0) i \leqq M \cdot m ! / \delta_{0}{ }^{n} \text { for any } x \in \prod_{k=1}^{n}\left\{s_{k}-\delta_{k} \leqq\left|x_{k}\right| \leqq s_{k}+\delta_{k}\right\} .
$$

This proves

$$
\left|\iota_{m}(x ; 0)\right| \leqq M \cdot m ! / \delta_{0}{ }^{m} \quad \text { for any } x \in \prod_{k=1}^{n}\left\{\left|x_{k}\right| \leqq s_{k}+\delta_{k}\right\} .
$$

This proves our lemma.

Q.E.D.

Proof of sufficiency in Theorem 2. It is now almost obvious from the above lemma. In fact, in the case where the coefficients of $J$ or $J^{-1}$ are singular at the origin, there exists $\left\{s_{k}\right\}_{k=0}^{n}$ such that the coefficients are holomorphic in a neighbourhood of $\prod_{k=1}^{n}\left\{\left|x_{k}\right|=s_{k}\right\} \times\left\{|t|=s_{0}\right\}$. On the other hand, we can choose $s_{k}$ as small as we need. This shows that $L$ is Kowalevskian at the origin.

Q.E.D.

Proof of Proposition 1. It is a result of the theorem of HamiltonCayley. By the definition of the weight $q$ of the characteristic polynomial $p(\zeta, \lambda)$ of $L$, theie exists a characteristic root $\lambda\left(\tau \cdot \zeta_{0}\right)$ of $P\left(\tau \cdot \zeta_{0}\right)$ satisfying

$$
\lambda\left(\tau \zeta_{0}\right)=O\left(\tau^{q}\right), \quad \tau \rightarrow \infty .
$$

Since $\lambda\left(\tau \zeta_{0}\right)^{m}=O\left(\tau^{m q}\right)$ is a characteristic root of $P\left(\tau \zeta_{0}\right)^{m}$, it is obvious 
that $\lim \inf$ order $P\left(\partial_{x}\right)^{m} / m \geqq q$. Next, by the theorem of HamiltonCayley we obtain

$$
P(\zeta)^{N+k}=\sum_{j=1}^{N} a_{j}^{(k)}(\zeta) P(\zeta)^{N-j}, \quad k=0,1, \cdots
$$

where $a_{j}^{(0)}=a_{j}, \quad(j=1,2, \cdots, N)$ and $p(\zeta, \lambda)=\lambda^{N}-\sum_{j=1}^{N} a_{j}(\zeta) \lambda^{N-j}$. It is easy to see that $\left\{a_{j}^{(k)}\right\}_{j=1}^{N}$ satisfies the following asymptotic formula.

$$
\left(\begin{array}{c}
a_{1}^{(k)} \\
a_{2}^{(k)} \\
\cdot \\
\cdot \\
\cdot \\
a_{N}^{(k)}
\end{array}\right)=\left(\begin{array}{cccccc}
a_{1} & 1 & & & \\
a_{2} & & & & & \\
\cdot & & & \cdot & \\
\cdot & 0 & & \cdot & \\
\cdot & & & & \cdot \\
a_{N} & & & & & 0
\end{array}\right)\left(\begin{array}{c}
a_{1}^{(k-1)} \\
a_{2}^{(k-1)} \\
\cdot \\
\cdot \\
\cdot \\
a_{N}^{(k-1)}
\end{array}\right) .
$$

(3. 10) implies immediately that $\operatorname{deg} a_{j}^{(k)} \leqq(j+k) q, j=1,2, \cdots, N$, that is, order $a_{j}^{(k)}\left(\partial_{x}\right) P\left(\partial_{x}\right)^{N-j} \leqq(j+k) q+(N-j) p$, where $T$ is an admissible system of $P$. This shows that

$$
\lim _{m \rightarrow \infty} \sup \operatorname{order} P\left(\partial_{x}\right)^{m} / m \leqq q .
$$

Before the proof of Theorem 3, we shall prepare the following lemma.

Lemma 3. 3. Let $P=P\left(x ; \partial_{x}\right)$ and $J\left(x ; \partial_{x}\right)$ satisfy the conditions (i) and (ii) in Theorem 2. Then we have

$$
\limsup _{m \rightarrow \infty} \operatorname{order} P^{m} / m=\limsup _{m \rightarrow \infty} \operatorname{order} J^{-1} P^{m} J / m \text {. }
$$

Proof. Let order $P^{m}=p(m), T_{m}=\left\{t_{i}^{(m)}\right\}_{i=1}^{N}$ be an admissible system of $P^{m}$ and $r(\in \boldsymbol{Q})$ be sufficiently large constant such that $\underset{T_{m}}{\operatorname{order}} J \leqq r$, $\underset{T_{m}}{\operatorname{order}} J^{-1} \leqq r$ and also order $P^{q} \leqq r$ for any $q=0,1, \cdots, m-1 .{ }_{T_{m}}^{T_{m}}$ Then if we put $l=s m+q,(q=0,1, \cdots, m-1)$, it follows that

$$
\underset{T_{m}}{\operatorname{order}} J^{-1} P^{l} J \leqq s p(m)+3 r
$$

which implies

(3. 12) $\quad \lim _{m \rightarrow \infty} \sup$ order $J^{-1} P^{m} J / m \leqq \lim _{m \rightarrow \infty} \sup$ order $P^{m} / m$.

On the other hand, the inverse inequality is now obvious. Q.E.D. 
Proof of Theorem 3. First, we remark that if $\limsup _{m \rightarrow \infty}$ order $P^{m} / m$ <order $P$, then the principal part $\stackrel{\circ}{P}(x ; \zeta)$ is nilpotent for any $(x, \zeta)$. Then there exists an operator $J$ satisfying the condition in Theorem 3, that is, order $J^{-1} P J=q$, where $q$ is the one defined by (1.13). Moreover the principal part of $J^{-1} P J$ is not nilpotent.

Now it is sufficient to prove the necessity, since the sufficiency is proved in Theorem 2 .

Proof of Neccssity. Its proof is some modification of that of Theorem 1. Under the condition that $q>1$, it is obvious that $L$ is not Kowalevskian at the origin if the coefficients of $J$ and $J^{-1}$ are holomorphic at the origin. Therefore, it suffices to consider the case where the coefficients of $J$ or $J^{-1}$ have pole at the origin and $q>1$. Under the assumption that $L$ is Kowalevskian at the origin, we know that there exists a domain $D$ such that the Cauchy problem $P u=0,\left.u\right|_{t=0}=U(x) \in H\left(\mathcal{O}_{x}\right)$ has a holomorphic solution $u \in H(D)$. Now without loss of generality, we may assume that the coefficients of $J$ or $J^{-1}$ have pole only at the origin and assume that the order of pole is at most $k$. Let $j=\max _{i, j}$ \{order $\left.j_{i j}\left(x ; \partial_{x}\right)\right\}$, where $J=\left(j_{i j}\right)$, and let $\left(x_{0}, \zeta_{0}\right)$ be a point in $\left(D_{\cap}\{t=0\}\right) \times \mathbb{C}^{1}$ such that the principal part of $J^{-1} P J$ iu Volevič's sense has non-zero characteristic values at $\left(x_{0}, \zeta_{0}\right)$. Under the above conditions, let us consider the following Cauchy problem,

$$
\begin{aligned}
& \partial_{t} v=J^{-1} P J v, \\
& \left.v\right|_{t=0}=x^{k+j} V(x), \quad V(x) \in H\left(\mathcal{O}_{x}\right) .
\end{aligned}
$$

Then in view of the proof of Theorem 1, we can construct $V(x)$ $\in H\left(\mathcal{O}_{x}\right)$ such that the Cauchy problem (3.13)-(3.14) has not holomorphic solution at $\left(x_{0}, 0\right)$. This contradicts the assumption that $L$ is Kowalevskian at the origin. In fact, the above Cauchy problem is equivalent with the following,

$$
\begin{aligned}
& \partial_{t}(J v)=P(J v), \\
& \left.J v\right|_{t=0}=J\left(x^{k+j} V(x)\right) \in H\left(\mathcal{O}_{x}\right) .
\end{aligned}
$$

At the end of this section, we shall consider the following example defined in $\mathbb{C}_{x, t}^{n+1}$ which can not be applied the above method, 


$$
P\left(x, t ; \partial_{x}\right)=a(x, t)\left[\begin{array}{cc}
\partial_{x_{1}} \partial_{x_{2}} & \partial_{x_{1}}^{2} \\
-\partial_{x_{2}}^{2} & -\partial_{x_{1}} \partial_{x_{2}}
\end{array}\right]+Q\left(x, t ; \partial_{x}\right),
$$

where $Q=\left(q_{i j}\right)$, order $q_{i j} \leqq 1$ and $a(x, t) \in H(\mathcal{O})$. Then the system $L=\partial_{t} I_{2}-P$ is Korvalevskian at every point in $\mathcal{O}$ if and only if

$$
Q\left(x, t ; \partial_{x}\right)=\left[\begin{array}{ll}
\alpha \partial_{x_{1}}+\beta \partial_{x_{2}}+g\left(x, t ; \partial_{x}\right)+a_{11}, & \beta \partial_{x_{1}}+\gamma \partial_{x_{1}}+a_{12} \\
-\alpha \partial_{x_{2}}+\delta \partial_{x_{2}}+a_{21}, & \delta \partial_{x_{1}}-\gamma \partial_{x_{2}}+g\left(x, t ; \partial_{x}\right)+a_{22}
\end{array}\right]^{2)}
$$

where $\alpha, \beta, \cdots, a_{i j} \in H(\mathcal{O})$ and $g\left(x, t ; \partial_{x}\right)$ is a homogeneous operator of order 1 .

First, we shall show the necessary condition. Let

$$
\widetilde{P}=\left[\begin{array}{ccc}
p_{11} & p_{12} & a \partial_{x_{1}} \\
p_{21} & p_{22} & -a \partial_{x_{2}} \\
0 & 0 & 0
\end{array}\right], \quad \text { order } \widetilde{P}=\operatorname{order} P .
$$

Then it is easy to see that $L$ is Kowalevskian if and only if $\widetilde{L}=\partial_{t} I_{3}-\widehat{P}$ is Kowalevshian. Let

$$
J\left(\partial_{x}\right)=\left[\begin{array}{ccc}
1 & & \\
& 1 & \\
-\partial_{x_{2}} & -\partial_{x_{1}} & 1
\end{array}\right], \quad J^{-1}\left(\partial_{x}\right)=\left[\begin{array}{ccc}
1 & & \\
& 1 & \\
\partial_{x_{2}} & \partial_{x_{1}} & 1
\end{array}\right] .
$$

Then we have

$$
\begin{aligned}
J^{-1} L J & =\partial_{t} I_{3}-\left[\begin{array}{ccc}
q_{11} & q_{12} & a \partial_{x_{1}} \\
q_{21} & q_{22} & -a \partial_{x_{2}} \\
\partial_{x_{2}} q_{11}+\partial_{x_{1}} q_{21}, & \partial_{x_{2}} q_{12}+\partial_{x_{1}} q_{22}, & \partial_{x_{2}}\left(a \partial_{x_{1}}\right)-\partial_{x_{1}}\left(a \partial_{x_{2}}\right)
\end{array}\right], \\
& =\partial_{t} I_{3}-\tilde{P},
\end{aligned}
$$

where order $\tilde{\widetilde{P}} \leqq 3 / 2$. Now let $\stackrel{\circ}{q}_{i j}$ be the homogeneous part of order 1 of $q_{i j}$. Then order $P<3 / 2$ implies immediately that

$$
\dot{\circ}_{11}=\alpha \partial_{x_{1}}, \quad \stackrel{\circ}{q}_{21}=-\alpha \partial_{x_{2}}, \quad \stackrel{\circ}{q}_{12}=\gamma \partial_{x_{1}}, \quad \stackrel{\circ}{q}_{22}=-r \partial_{x_{2}} .
$$

On the other hand, if order $\tilde{\widetilde{P}}=3 / 2$ and $L$ is Kowalevskian, then it holds that

$$
\begin{aligned}
& \left\{\stackrel{\circ}{q}_{11}(x, t ; \zeta) \zeta_{2}+\stackrel{\circ}{q}_{21}(x, t ; \zeta) \zeta_{1}\right\} \zeta_{1} \\
& \quad-\left\{\stackrel{\circ}{q}_{12}(x, t ; \zeta) \zeta_{2}+\stackrel{\circ}{q}_{22}(x, t ; \zeta) \zeta_{1}\right\} \zeta_{2} \equiv 0 .
\end{aligned}
$$

2) In the case where $P=P\left(x, \partial_{x}\right)$, we can show that (1.12) is valid if and only if $Q(x$; $\partial_{x}$ ) has the form (3.18). 
From (3.21) we have the condition (3.18) immediately.

Next, we shall prove the sufficient condition. More pricisely, we shall show that under the condition (3.18) we can reduce the system $L=\partial_{t} I_{2}-P$ to the equivalent Kowalevskian system in Volevič's sense. Let $g\left(x, t ; \partial_{x}\right)=A(x, t) \partial_{x_{1}}+B(x, t) \partial_{x_{2}}+\widetilde{g}\left(x, t ; \partial_{\tilde{x}}\right)$, where $\partial_{\widetilde{x}}=\left(\partial_{x_{3}}, \cdots\right.$, $\left.\partial_{x_{n}}\right)$, and let

$$
h=\left[\begin{array}{ccc}
p_{11}, & p_{12}, & a \partial_{x_{1}}+\beta+B \\
p_{21}, & p_{22}, & -a \partial_{x_{2}}+\delta+A \\
0 & 0 & 0
\end{array}\right]^{3)}, \quad \text { order } h=\operatorname{order} P
$$

Then we have

$J^{-1} \mathcal{L} J=\partial_{t} I_{3}-\left[\begin{array}{ccc}A \partial_{x_{1}}+\alpha \partial_{x_{1}}+\widetilde{g}+a_{11}, & -B \partial_{x_{1}}+\gamma \partial_{x_{1}}+a_{12}, & a \partial_{x_{1}}+\beta+B \\ -A \partial_{x_{2}}-\alpha \partial_{x_{2}}+a_{21}, & B \partial_{x_{2}}-\gamma \partial_{x_{2}}+\widetilde{g}+a_{22}, & -a \partial_{x_{2}}+\delta+A \\ \widetilde{h}_{31}, & \widetilde{\not}_{32}, & \widetilde{\not}_{33}\end{array}\right]$,

where $\mathcal{L}=\partial_{t} I_{3}-\mathcal{L}$ and

$$
\begin{aligned}
& \widetilde{h}_{31}=\partial_{x_{2}}\left\{A \partial_{x_{1}}+\alpha \partial_{x_{1}}+\widetilde{g}+a_{11}\right\}+\partial_{x_{1}}\left\{-A \partial_{x_{2}}-\alpha \partial_{x_{2}}+a_{21}\right\}, \\
& \widetilde{h}_{32}=\partial_{x_{2}}\left\{-B \partial_{x_{1}}+\gamma \partial_{x_{1}}+a_{12}\right\}+\partial_{x_{1}}\left\{B \partial_{x_{2}}-\gamma \partial_{x_{2}}+\widetilde{g}+a_{22}\right\}, \\
& \widetilde{h}_{33}=\partial_{x_{2}}\left\{a \partial_{x_{1}}+\beta+B\right\}+\partial_{x_{1}}\left\{-a \partial_{x_{2}}+\delta+A\right\} .
\end{aligned}
$$

Therefore if $\tilde{g}\left(x, t ; \partial_{\tilde{x}}\right) \equiv 0$, then $J^{-1} \mathcal{L} J$ is Kowalevskian in Volevič's sense. So let us consider the case where $\widetilde{g} \not \equiv 0$. Let $\widetilde{f}\left(x, t ; \partial_{x}\right)=J^{-1} \not J J$ $=\left(\tilde{h}_{i j}\right)_{i, j=1,2,3}$, and let

$$
\tilde{h}=\left(\begin{array}{cccc}
\tilde{h}_{11} & \widetilde{h}_{12} & \widetilde{h}_{13} & 0 \\
\widetilde{h}_{21} & \widetilde{h}_{22} & \widetilde{h}_{23} & 0 \\
\widetilde{h}_{31} & \widetilde{h}_{32} & \widetilde{h}_{33} & \tilde{g} \\
0 & 0 & 0 & \tilde{g}
\end{array}\right), \quad \tilde{J}\left(\partial_{x}\right)=\left(\begin{array}{cccc}
1 & & & \\
& 1 & & \\
& & 1 & \\
-\partial_{x_{2}} & -\partial_{x_{1}} & 0 & 1
\end{array}\right) .
$$

Then it is easy to see that $L$ is Kowalevskian if and only if $\tilde{\mathcal{L}}=\partial_{t} I_{4}-\tilde{\mathscr{C}}$ is Kowalevskian, because of the fact that order $\widetilde{g}=1$. By an elementary calculation, we have

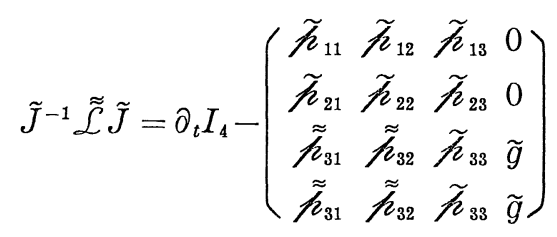

s) It is easy to see that order $P^{m}=\operatorname{order} \hbar^{m}$ for any $m \geqq 1$. 
where $\widetilde{h}_{31}=\widetilde{g} \partial_{x_{2}}+\tilde{h}_{31}, \widetilde{h}_{32}=\tilde{g} \partial_{x_{1}}+\tilde{h}_{32}$ and order $\tilde{h}_{3 j} \leqq 1, j=1,2$. Thus we have proved that $L$ is Kowalevskian. The proof of $\lim$ order $P^{m} / m$ $=1$ is easy in the case where $g \not \equiv 0$.

\section{$\S$ 4. Proof of Theorem}

In this section, $z$ and $x$ denote $z=\left(z_{1}, \cdots, z_{n}\right) \in \boldsymbol{C}^{n}$ and $x=\left(x_{1}, \cdots, x_{n}\right)$ $\in \boldsymbol{R}^{n}$ respectively, and $z_{0} \in \mathbb{C}^{1}$ and $t \in \mathbb{R}^{1}$.

First, let us consider the Cauchy problem,

$$
\begin{aligned}
& L\left(z, z_{0} ; \partial_{z}, \partial_{z_{0}}\right) u=0, \\
& \left.u\right|_{z_{0}=0}=U(z),
\end{aligned}
$$

where $L$ has an operator $J$ satisfying the conditions (i), (ii) and (iii) in Theorem 2 and the coefficients of $L$ are holomorphic in $\mathcal{O}$ given in section 1 .

From Theorem 2 we know that $L$ is Kowalevskian at every point in $\mathcal{O}$. Now let $U(z) \in H(\omega)$ where $\omega \subset \mathcal{O}_{z},\left(\mathcal{O}_{z}=\mathcal{O}_{\cap}\left\{z_{0}=0\right\}\right)$. Then for any $J \Subset \omega$ there exists $s_{0}(>0)$ such that the solution $u$ of $(4.1)-(4.2)$ exists in $J \times\left\{\left|z_{0}\right| \leqq s_{0}\right\}$ and moreover there exists $K(J \mathbb{C} K \Subset \omega)$ such that

$$
\sup _{J \times\left\{\left|z_{0}\right| \leqq s_{0}\right\}}|u| \leqq C \sup _{K}|U|,
$$

where $C$ depends only on $L$.

Its proof is done by the same way as that of Theorem 2, considering the remark before Lemma 3.2. Now we can choose $s_{0}$ as small as the Cauchy problem for (4.1) with Cauchy data

$$
\left.u\right|_{z_{0}=\tau_{0}}=U(z), \quad\left(\left|\tau_{0}\right| \leqq s_{0}\right)
$$

has also a solution in $J \times\left\{\left|z_{0}-\tau_{0}\right| \leqq s_{0}\right\}$ and the similar inequality with (4.3) holds with constant $C$ which is chosen uniformly in $\tau_{0}$ (see the proof of Lemma 3.2).

Now let the coefficients of $L\left(x, t ; \partial_{x}, \partial_{t}\right)$ be real analytic in $\Omega \subset \mathbb{R}^{n+1}$, $(0 \in \Omega)$. Assume that these coefficients can be holomorphic extension to $\Omega_{a}=\left\{\left(z, z_{0}\right) \in C^{n+1} ;\left|z_{k}-x_{k}\right|<a,\left|z_{0}-t\right|<a, k=1, \cdots, n,(x, t) \in \Omega\right\} . \quad$ It is evident that $L\left(z, z_{0} ; \partial_{z}, \partial_{z_{0}}\right)$ has the same properties in Theorem 4 . 
Adjoint operator ${ }^{t} L$ of $L$ has also the same properties in Theorem 4 . In fact, ${ }^{t}\left(J^{-1} L J\right)={ }^{t} J^{t} L^{t}\left(J^{-1}\right)={ }^{t} J^{t} L\left({ }^{t} J\right)^{-1}$.

Now our purpose is to prove that if $\iota \in C^{1}\left([-T, T] ; \mathscr{D}^{\prime}\left(\Omega_{x}\right)\right)$ satisfy the Cauchy problem,

$$
\begin{aligned}
& L_{u}=0, \\
& u(x, 0)=0,
\end{aligned}
$$

then $u$ vanishes in a neighbourhood of the origin.

Let $\rho(x) \in C_{0}^{\infty}\left(\Omega_{x}\right)$, where $\rho=1$ on $W\left(0 \in W \subset \Omega_{x}\right)$, and let $w$ $=\rho u$. hen we have

$$
L w=f,
$$

$$
w(x, 0)=0,
$$

where $f=\rho(x) P\left(x, t ; \partial_{x}\right) u-P\left(x, t ; \partial_{x}\right) \rho(x) u \in C^{0}\left([-t, T] ; \mathscr{D}^{\prime}\left(\Omega_{x}\right)\right)$ and supp $f \subset \operatorname{supp}\left[\rho_{x}(x)\right]$ for any $t \in[-T, T]$, where $\rho_{x}=\operatorname{grad} \rho(x)$. In the following, we put supp $\left[\rho_{x}\right]=V$.

Now let $v\left(z, z_{0} ; t_{0}\right)$ be a solution of the Cauchy problem,

$$
\begin{aligned}
& { }^{t} L\left(z, z_{0} ; \partial_{z}, \partial_{z_{0}}\right) v=0, \\
& \left.v\right|_{z_{0}=t_{0}}={ }^{t}(0, \cdots, \underset{\widehat{\kappa}}{(z)}, 0, \cdots, 0), \quad\left(0<t_{0} \in \mathbb{R}\right) .
\end{aligned}
$$

where $\phi(z) \in H\left(\bar{V}_{b}\right), \bar{V}_{b}=\left\{z \in \mathbb{C}^{n} ;\left|z_{k}-x_{k}\right| \leqq b, k=1, \cdots, n, x \in V\right\}, b<a$.

From the considerations in the above, we can see that for any $c(c<b)$ there exists $t_{0}$ sufficiently small which depends on $b$ and $c$, such that the solution $v\left(z, z_{0} ; t_{0}\right)$ exists in a neighbourhood of $V_{c} \times\left[0, t_{0}\right]$, and the following inequality holds,

$$
\sup _{\bar{V}_{c} \times\left[0, t_{0}\right]}\left|v\left(z, z_{0} ; t_{0}\right)\right| \leqq C \sup _{\bar{V}_{b}} \mid \phi(z) !,
$$

where $C$ depends only on $L$.

Under these considerations, we prove our theorem. (4.8)-(4.9) is equivalent with

$$
v\left(z, t ; t_{0}\right)=v\left(z, t_{0} ; t_{0}\right)-\int_{t_{0}}^{t} P\left(z, \tau ; \partial_{z}\right) v\left(z, \tau ; t_{0}\right) d \tau,
$$

where $t \in \boldsymbol{R}$ and $v\left(z, t_{0} ; t_{0}\right)={ }^{t}(0, \cdots, \underset{\widehat{k}}{\phi(z)}, 0, \cdots, 0)$. Then we have 


$$
\begin{aligned}
\left\langle w\left(x, t_{0}\right), v\left(x, t_{0} ; t_{0}\right)\right\rangle= & \int_{0}^{t_{0}}\left\langle\partial_{t} w(x, t), v\left(x, t ; t_{0}\right)\right. \\
& \left.\quad+\int_{t_{0}}^{t} P v\left(x, \tau ; t_{0}\right) d \tau\right\rangle d t \\
= & \int_{0}^{t}\left\langle f(x, t), v\left(x, t ; t_{0}\right)\right\rangle d t .
\end{aligned}
$$

Thus we have

$$
\left\langle w_{k}\left(x, t_{0}\right), \phi(x)\right\rangle=\int_{0}^{t_{0}}\left\langle f(x, t), v\left(x, t ; t_{0}\right)\right\rangle d t .
$$

Let us now remark that $u \in \mathscr{D}^{\prime}(\bar{V})$, ( $\mathscr{D}^{\prime}(\bar{V})$ denotes the distribution with support in $\bar{V}$, where $\bar{V}$ is the closure of $V$ ) can be uniquely extended to analytic functional $H^{\prime}\left(\bar{V}_{c}\right)$ by the formula,

$$
\langle u, \phi\rangle_{H^{\prime}\left(\bar{V}_{c}\right) \times H\left(\bar{V}_{c}\right)}=\left\langle u,\left.\phi\right|_{\boldsymbol{R}^{n}}\right\rangle,
$$

where $H\left(\bar{V}_{c}\right)$ denotes the space obtained by complition of entire functions by the norm $\|u\|=\sup _{\bar{v}_{a}}|u|$.

In view of this remark, we have

$$
\begin{aligned}
\left|\left\langle w_{k}\left(x, t_{0}\right), \phi(x)\right\rangle\right| & \leqq C \max _{t \in\left[0, t_{0}\right]}\|f(z, t)\|_{H^{\prime}\left(\bar{V}_{c}\right)} \\
& \times \max _{t \in\left[0, t_{0}\right]}\left\|v\left(z, t ; t_{0}\right)\right\|_{H\left(\bar{V}_{c}\right)},
\end{aligned}
$$

for any $v\left(z, t ; t_{0}\right) \in H\left(\bar{V}_{c}\right)$. Now we define a sequence of entire functions $\left\{\phi^{(l)}(z)\right\}_{l=1}^{\infty}$ by

$$
\phi^{(l)}(z)=\left[\frac{l}{\sqrt{\pi}}\right]^{n} \int_{\boldsymbol{R}^{n}} h(y) \exp \left(-l^{2} \sum_{j=1}^{n}\left(z_{j}-y_{j}\right)^{2}\right) d y,
$$

$h(x) \in C_{0}^{\infty}(\omega), \omega \in \mathbb{E}$. We give now the constant $b$ as small as

$$
\sum_{j=1}^{n} \operatorname{Re}\left(z_{j}-y_{j}\right)^{2} \geqq \varepsilon>0, \quad \approx \in \bar{V}_{b}, \quad y \in \omega .
$$

Then by the determination of $b$ we obtain

$$
\begin{aligned}
& \phi^{(l)}(x) \rightarrow h(x) \quad \text { in } \mathcal{E}\left(\boldsymbol{R}^{n}\right), \\
& \phi^{(l)}(z) \rightarrow 0 \text { on } \bar{V}_{b} .
\end{aligned}
$$

We remark that if we choose $W$ sufficiently small, then the solutions $v^{(l)}$ corresponding to $\phi^{(l)}$ belong to $H\left(\bar{V}_{c}\right)$ for small $t_{0}$. Therefore by 
(4.10) and (4.14) we have

$$
\left|\left\langle w_{k}\left(x, t_{0}\right), \phi^{(l)}(x)\right\rangle\right| \leqq C^{\prime} \sup _{\bar{v}_{b}}\left|\phi^{(l)}(z)\right| .
$$

Hence we have $\left\langle w_{k}\left(x, t_{0}\right), h(x)\right\rangle=0$ for any $h(x) \in \mathbb{C}_{0}^{\infty}(\omega)$. Since $w$ $=\rho u$, this proves our theorem. Q.E.D.

\section{References}

[1] Gårding, L., Une variante de la méthod de majoration de Cauchy, Acta Math., 114 (1965), 143-158.

[2] Hasegawa, Y., On the initial value problems with data on a double characteristis, J. Math. Kyoto Univ., 11 (1971), 352-372.

[3] Kitagawa, K. and Sadamatsu, T., A necessary condition of Cauchy-Kowalevski's theorem, Publ. RIMS Kyoto Univ., 11 (1976), 523-534.

[4] Miyake, M., A remark on Cauchy-Kowalevski's Theorem, Publ. RIMS Kyoto Univ., 10 (1974), 243-255.

[5] Mizohata, S., On kowalevskian systems, Uspehi Mat. Nauk., 29 (1974), 216-227 (in Russian).

[6] - Cauchy-Kowalevski's theorem: A necessary condition, Publ. RIMS Kyoto Univ., 10 (1975), 509-519. 
